\title{
Etude d'un dosimètre individuel passif multi-élément pour la dosimétrie des neutrons *
}

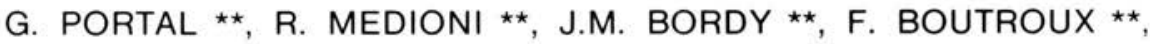

 \\ (Manuscrit reçu le 7 février 1986)
}

\begin{abstract}
RÉSUMÉ
La dosimétrie individuelle des neutrons est actuellement effectuée à partir de méthodes peu satisfaisantes, soit par l'utilisation d'émulsions nucléaires qui sont "aveugles" pour les énergies inférieures à $1,5 \mathrm{MeV}$, soit par la mise en œuvre de détecteurs à albédo qui doivent être étalonnés aux divers postes de travail car leur réponse varie considérablement en fonction de l'énergie des neutrons.

Les auteurs présentent l'état actuel des études du projet DINEM, Dosimètre Individuel "Neutrons" à Eléments Multiples. II est constitué d'un dosimètre à albédo "PGP-DIN" qui détecte les neutrons d'énergie inférieure à $7 \mathrm{keV}$ et d'un détecteur solide de traces qui détecte les énergies supérieures à $100 \mathrm{keV}$.

Les derniers progrès enregistrés sur l'utilisation du CN 85 comme détecteur solide de traces laissent entrevoir une solution prochaine au problème difficile de la dosimétrie individuelle.
\end{abstract}

\section{ABSTRACT}

Personal neutron dosimetry is presently carried out with unsatisfying methods, viz. the use of nuclear emulsions "blind" for energy below 1.5 MeV, or the operation of albedo detectors that must be calibrated at the various working places since their responses vary largely as a function of neutron energies. A progress report is presented on the DINEM project studies. The DINEM (personal multielement neutron dosimeter) is made of an albedo dosimeter "PGP-DIN" detecting neutrons with energies below $7 \mathrm{keV}$ and a solid track detector detecting energies above $100 \mathrm{keV}$. The latest improvements on the use of CN 85 as a solid track detector announce that the difficult problem of personal dosimetry should be solved soon.

* Communication présentée au congrès franco-italien "Les expositions profession. nelles, aspects méthodologiques et expériences pratiques", organisé par la Société française de radioprotection (SFRP) et l'Associazione italiana di radioprotezione (AIRP) à Nice, du 29 au 31 janvier 1986.

** CEA, IPSN/DPT/SIDR, BP 6, 92265 Fontenay-aux-Roses Cedex.

$\star \star \star$ Institut de dosimétrie des rayonnements, Académie des Sciences de Tchécoslovaquie, $\mathrm{Na}$ Truhlarce 39/64, 18086 Praha 8, Tchécoslovaquie. 


\section{INTRODUCTION}

Lors du congrès franco-italien qui s'est tenu à Florence du 30 mai au

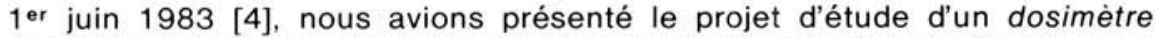
individuel neutrons à éléments multiples (DINEM). Nos études étaient alors consacrées à la mise en œuvre de deux types différents d'éléments détecteurs intégrateurs passifs : l'émulsion photographique et les détecteurs solides de traces. Depuis cette date, des progrès substantiels ont été réalisés sur la technologie des détecteurs solides de traces utilisés en France; tous nos efforts sont actuellement concentrés sur leur mise en œuvre.

Alors que dans la plupart des laboratoires étrangers les études sont consacrées à la mise au point d'un nouveau matériau (le CR 39), nous avons préféré réserver nos efforts à un matériau déjà utilisé depuis de nombreuses années pour la détection des alphas (neutronographie, détection du radon, etc.) : le CN 85.

Le CR 39 est un polycarbonate mis au point pour les besoins de l'optique ; il est fort séduisant car sa sensibilité est telle qu'il permet l'enregistrement de protons de forte énergie. Par contre, la mise au point de sa fabrication industrielle semble poser de réels problèmes de prix de revient et de reproductibilité des caractéristiques dosimétriques.

Le CN 85 est un nitrate de cellulose fabriqué depuis longtemps en France par Kodak Pathé. Sa sensibilité est inférieure à celle du CR 39 ; seuls les protons d'énergie inférieure à $1 \mathrm{MeV}$ peuvent être enregistrés ; il est difficile de distinguer leurs traces de celles du bruit de fond. Cependant, sa fabrication industrielle est beaucoup plus simple et son prix est environ dix fois inférieur.

Nous avons appliqué au CN 85 les méthodes de révélation des traces mises au point par L. TOMMASINO [6] pour le CR 39 : l'attaque électrochimique. Nous avons constaté, non sans une certaine surprise, que l'on obtient des résultats comparables à ceux du CR 39 et que, contrairement à ce qui a pu être publié à ce sujet, le CN 85 peut être aisément mis en $œ u v r e$ pour la détection des neutrons d'énergie supérieure à $100 \mathrm{keV}$.

\section{L'ATTAQUE ELECTROCHIMIQUE}

Cette étude étant réalisée dans le cadre d'une collaboration francotchécoslovaque, la mise au point de l'attaque électrochimique a été effectuée à I'IDR de Prague [5]. Le principe de la méthode est schématisé sur la figure 1. L'échantillon de CN 85 est placé dans un bain de soude et soumis à un champ électrique alternatif. On a constaté, avec un dispositif qui fournit un signal carré, que les conditions optimales d'attaque électrochimique sont les suivantes: 


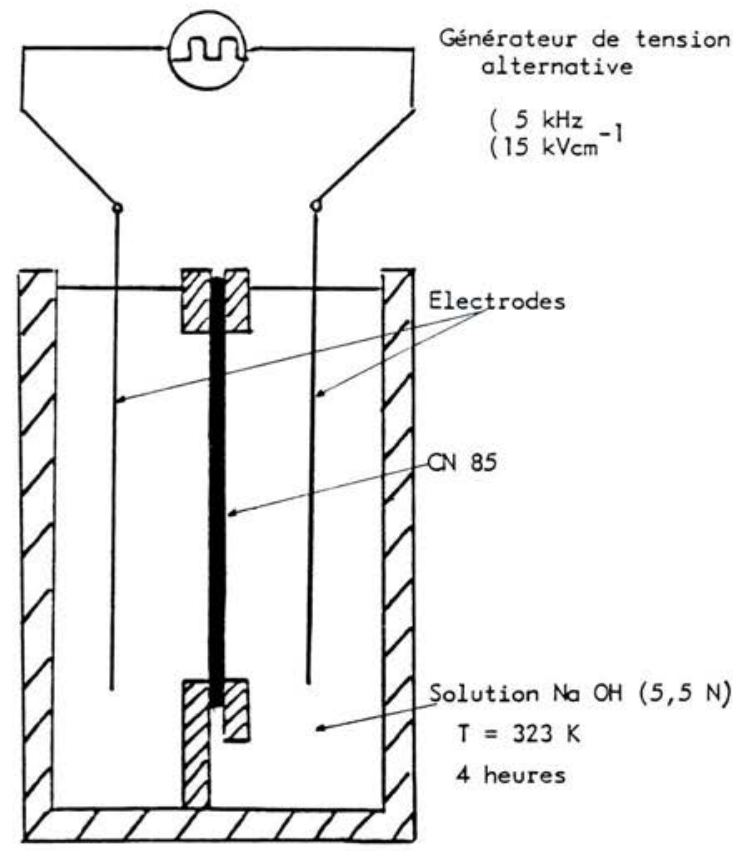

Fig. 1.- Principe d'une cellule à attaque électrochimique.

. concentration de la solution $\mathrm{NaOH}$ :

température de la solution :

fréquence :

champ électrique :

durée de l'attaque :
$5,5 \mathrm{~N}$

$323 \mathrm{~K}$

$5 \mathrm{kHz}$

$15 \mathrm{kV} . \mathrm{cm}^{-1}$

$4 \mathrm{~h}$

Dans ces conditions les traces révélées sont visibles avec un faible grossissement et peuvent être distinguées de celles du bruit de fond avec un minimum de confusion.

\section{LE COMPTAGE DES TRACES}

Les études ont été effectuées avec un microscope optique. Nous avons utilisé un grossissement de 250 , ce qui est faible si on le compare à celui utilisé pour l'analyse des émulsions nucléaires NTA actuellement utilisées pour la surveillance opérationnelle du personnel.

La lecture des densités moyennes ou fortes de traces est effectuée à l'aide d'un analyseur automatique de champs (Analyseur d'images 40-10 de Systèmes analytiques). Cet appareil permet de programmer la taille mini- 
male des traces prises en compte par l'appareil et donc facilite la sélection des traces de protons de celles du bruit de fond. Le dénombrement d'un champ est très rapide ; sa surface est réduite : $0,4 \mathrm{~mm}^{2}$ pour un grossissement de 100 .

La lecture des faibles densités de traces, et notamment la sélection des dosimètres irradiés, peut être effectuée plus rapidement à l'aide d'un lecteur de microfiche (grossissement 50 environ). On peut effectivement analyser une grande surface en un seul champ $\left(\sim 0,25 \mathrm{~cm}^{2}\right)$.

\section{REPONSE EN FONCTION DE L'ENERGIE}

L'étude de la réponse d'un dosimètre, constitué d'une feuille de CN 85 de $100 \mu \mathrm{m}$ d'épaisseur placée derrière un convertisseur hydrogéné en polyéthylène de $1 \mathrm{~mm}$ d'épaisseur, a été effectuée lors d'irradiations réalisées dans le cadre d'un groupe de travail européen : le CENDOS (collection and evaluation of data for neutron dosimetry).

Les dosimètres étaient irradiés, pour cette expérience préliminaire, à l'air libre sous une incidence normale. Les rayonnements utilisés provenaient de sources monochromatiques $(0,12-0,57-1,5-2,8-5,3-14 \mathrm{MeV})$, d'un empilement thermique et de sources isotopiques à spectre étendu (Am- $\mathrm{Li}$, $\mathrm{Cf} \mathrm{nu}, \mathrm{Cf}$ dans une sphère d'eau lourde de $30 \mathrm{~cm}$ de diamètre). Les essais avec un fantôme seront réalisés à la fin de l'étude sur un dosimètre plus proche de la version finale.

On constate sur la figure 2 les phénomènes suivants :

- Pour le domaine d'énergie concernant la radioprotection courante, c'est-à-dire pour les énergies inférieures à $3 \mathrm{MeV}$, la réponse du détecteur au CN 85 varie de \pm 40 pour cent autour d'une valeur moyenne. Cette dépendance énergétique n'est pas exagérée car les spectres réels que l'on rencontre en radioprotection sont très étalés en énergie et l'on obtient ainsi une compensation de la réponse globale qui atténue fortement les variations de réponse.

A titre d'exemple nous avons calculé la réponse globale pour deux configurations spectrales très différentes déduites de nos mesures effectuées respectivement dans un atelier de technologie de plutonium et dans le hall du réacteur belge de Tihange (figure 3 , d'après [1]).

Les réponses moyennes de ce type de dosimètre sont respectivement: . atelier $\mathrm{Pu}$

- réacteur de Tihange $\mathrm{R}=230$ traces $\mathrm{cm}^{-2} \cdot \mathrm{mSv}^{-1}$

Si l'on considère que ces deux cas représentent des situations extrêmes en radioprotection, on conçoit l'intérêt de cette technique.

- Pour les énergies supérieures, on observe un défaut de réponse entre 3 et $7 \mathrm{MeV}$. Ce défaut est dû essentiellement à la configuration expérimentale choisie. Cette réponse peut être améliorée en utilisant une feuille de CN 85 plus épaisse $(200 \mu \mathrm{m}$ au lieu de $100 \mu \mathrm{m})$ et en adoptant un convertisseur hydrogéné "équilibré". Un tel dispositif est réalisable mais ne présente d'intérêt que pour des postes de travail très particuliers. 


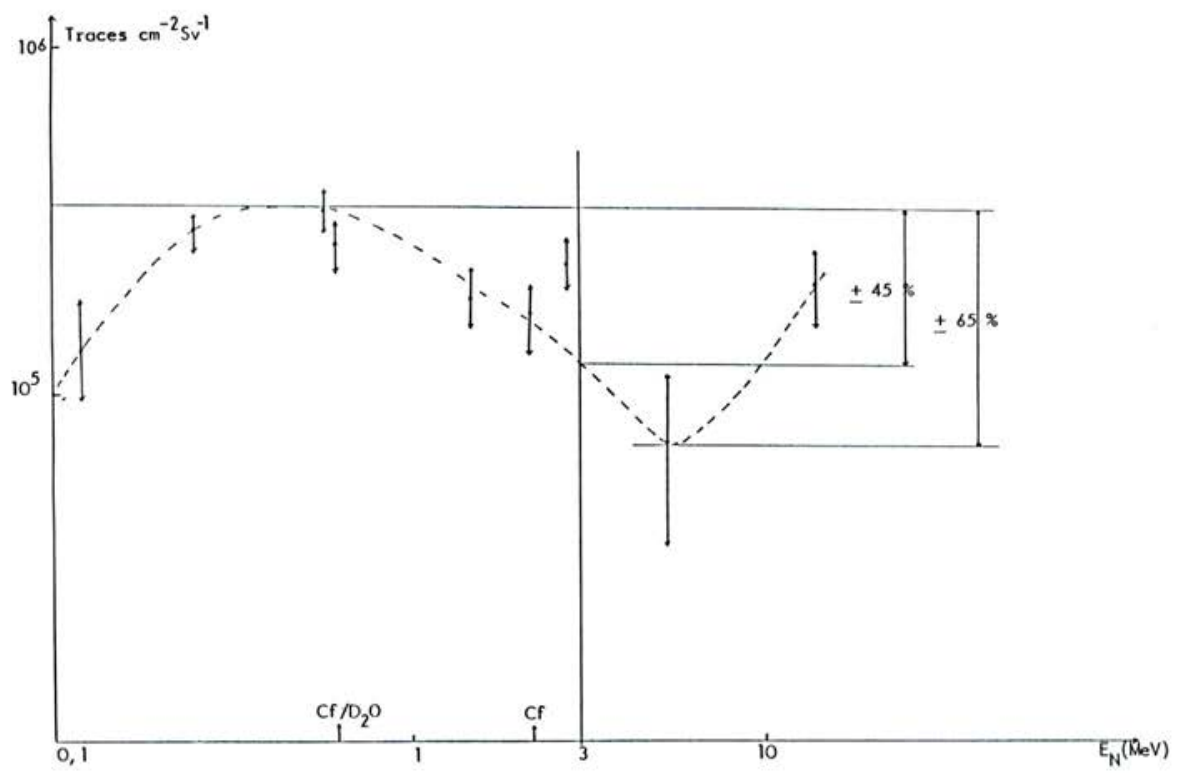

Fig. 2.- Réponse en fonction de l'énergie des neutrons du détecteur: $\mathrm{CN} 85+1 \mathrm{~mm}\left(\mathrm{CH}_{2}\right)_{n}$.

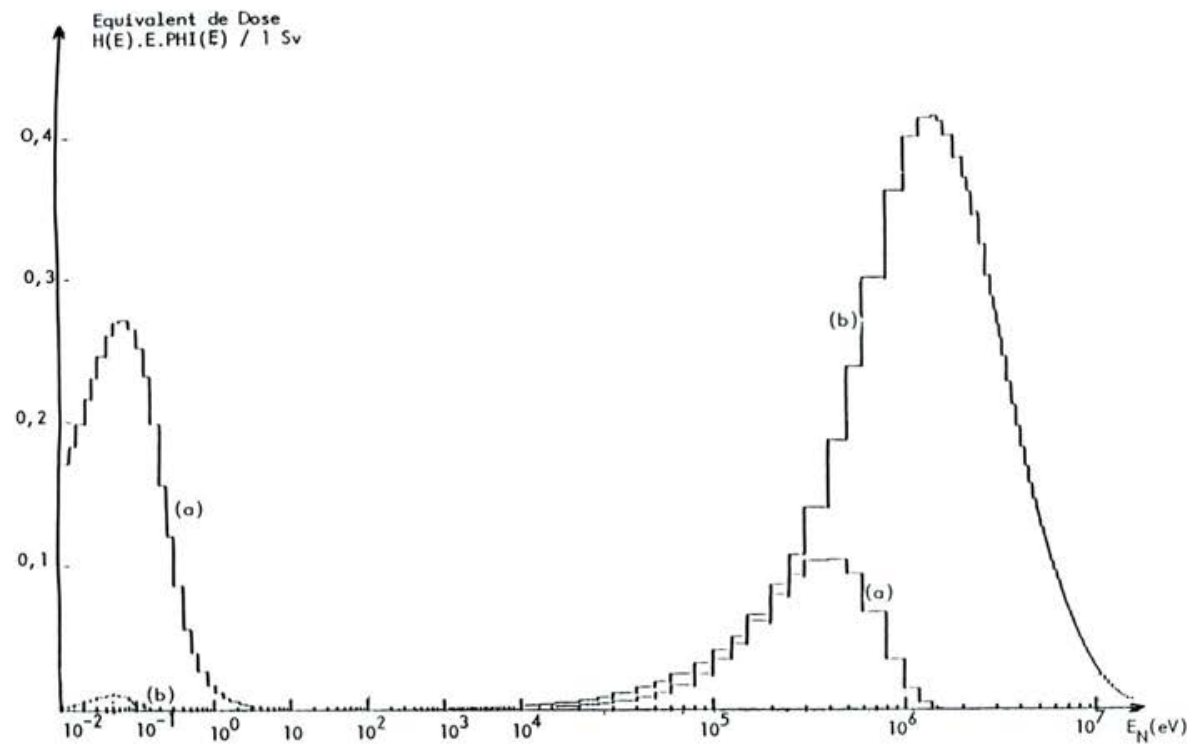

Fig. 3.- Spectres (a) Tihange niveau intermédiaire, (b) Pu Métal, d'après [1]. 


\section{LE DOSIMETRE DINEM}

A partir de ces résultats nous envisageons une première version très simple du dosimètre DINEM en accouplant au détecteur CN 85 le dosimètre à albédo PGP-DIN thermoluminescent qui est actuellement en service au CEA [2]. Ce dosimètre est recouvert d'un filtre de cadmium d'épaisseur calibrée $(0,1 \mathrm{~mm})$, ce qui lui confère une réponse indépendante de l'énergie pour les neutrons d'énergie inférieure à $7 \mathrm{keV}$.

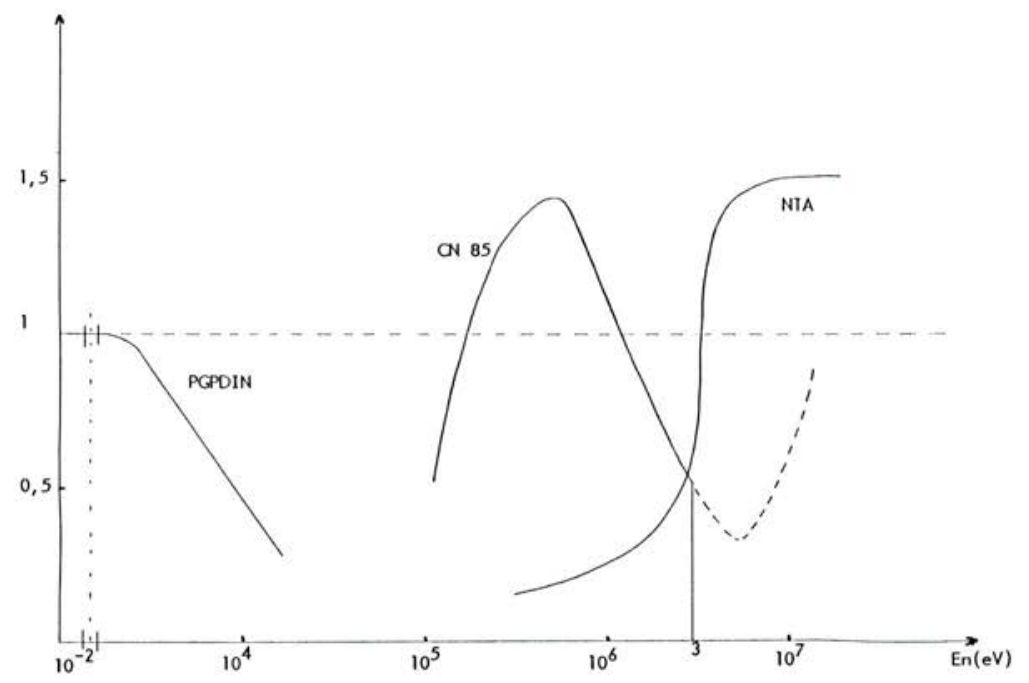

Fig. 4.- Réponse du dosimètre DINEM comparée à celle du NTA [6].

La courbe de réponse en énergie d'un tel dosimètre serait similaire à celle de la figure 4 obtenue par addition des deux courbes de réponse précédentes (CN 85 et PGP-DIN). On constate que la zone "aveugle" laissée par le dosimètre à émulsion nucléaire NTA est fortement réduite avec le détecteur à $\mathrm{CN} 85$. II est raisonnable de penser que l'on pourra "couvrir" cette zone "aveugle" par un artifice simple tel que le calcul basé sur des spectres modèles. Les premiers résultats montrent que le seuil de détection de ce dosimètre devrait être inférieur à 40 mrem $(0,4 \mathrm{mSv})$, seuil actuellement obtenu avec le dosimètre NTA.

II est intéressant de remarquer que la lecture du dosimètre à albédo thermoluminescent, lecture effectuée à l'aide d'un lecteur automatique rapide, permettra de sélectionner les détecteurs au CN 85 qui devront être "révélés" et "lus".

On disposera, enfin, d'un premier modèle opérationnel de dosimètre individuel pour les neutrons qui ne nécessitera pas un étalonnage auprès de chaque poste de travail comme c'est le cas pour les détecteurs à albédo actuellement utilisés. 


\section{REMERCIEMENTS}

Ces études ont été partiellement financées par le contrat BIO-A-543-83-F de la Commission des Communautés européennes.

\section{BIBLIOGRAPHIE}

[1] BUXEROLLE M., CERUTTI M., KURDJIAN J. Catalogue de spectres de neutrons (communication personnelle, Centre d'études nucléaires de Cadarache).

[2] BUXEROLLE M. Information sur le PGP-DIN, dosimètre individuel de neutrons. Rapport DPT/SIDR/GDN, Centre d'études nucléaires de Cadarache, 1985.

[3] HOFERT M., PIESCH E. Neutron dosimetry with nuclear emulsions. Radiat. Prot. Dos., $1985,10(1 / 4)$ 189-195.

[4] PORTAL G., MEDIONI R. Perspectives concernant la dosimétrie individuelle des neutrons au Commissariat à l'énergie atomique. Congrès commun italo-français de radioprotection, Firenze, Italie, 30 mai-1 er juin 1983.

[5] SPURNY F., BORDY J.M., MEDIONI R., PORTAL G., TUREK K. Development of fast neutron detector for multielement personal neutron track dosemeter. 13. Conférence internationale sur les détecteurs de traces à l'état solide, Rome, 23-27 septembre 1985.

[6] TOMMASINO L., ZAPPAROLI G., SPIEZIA P., GRIFFITH R.V., ESPINOSA G. Different etching processes of damage track detectors for personnel neutron dosimetry. 12. International conference on solid state nuclear track detectors, Acapulco, Mexico, 4-10 September 1983. Nucl. Tracks, 1984, 8(1/4) 335-339. 